\title{
Immune Response to Mycobacterium tuberculosis: A Narrative Review
}

\author{
Maurizio de Martino, Lorenzo Lodi, Luisa Galli and Elena Chiappini* \\ Department of Health Sciences, University of Florence, Florence, Italy
}

The encounter between Mycobacterium tuberculosis (Mtb) and the host leads to a complex and multifaceted immune response possibly resulting in latent infection, tubercular disease or to the complete clearance of the pathogen. Macrophages and $\mathrm{CD}^{+} \mathrm{T}$ lymphocytes, together with granuloma formation, are traditionally considered the pillars of immune defense against Mtb and their role stands out clearly. However, there is no component of the immune system that does not take part in the response to this pathogen. On the other side, Mtb displays a complex artillery of immune-escaping mechanisms capable of responding in an equally varied manner. In addition, the role of each cellular line has become discussed and uncertain further than ever before. Each defense mechanism is based on a subtle balance that, if altered, can lean to one side to favor Mtb proliferation, resulting in disease progression and on the other to the host

OPEN ACCESS

Edited by:

Gian Luigi Marseglia,

Policlinico San Matteo Fondazione

(IRCCS), Italy

Reviewed by:

Silvia Garazzino,

University Hospital of the City of Health and Science of Turin, Italy

Sara Manti,

University of Catania, Italy

*Correspondence:

Elena Chiappin

elena.chiappini@unifi.it

Specialty section:

This article was submitted to

Pediatric Immunology,

a section of the journal

Frontiers in Pediatrics

Received: 07 April 2019 Accepted: 06 August 2019

Published: 27 August 2019

Citation:

de Martino M, Lodi L, Galli L and Chiappini E (2019) Immune Response to Mycobacterium tuberculosis: A

Narrative Review.

Front. Pediatr. 7:350

doi: 10.3389/fped.2019.00350 tissue damage by the immune system itself. Through a brief and complete overview of the role of each cell type involved in the Mtb response, we aimed to highlight the main literature reviews and the most relevant studies in order to facilitate the approach to such a complex and changeable topic. In conclusion, this narrative mini-review summarizes the various immunologic mechanisms which modulate the individual ability to fight Mtb infection taking in account the major host and pathogen determinants in the susceptibility to tuberculosis.

Keywords: Mycobacterium tuberculosis, tuberculosis, children, immune response, immunity, macrophage, adaptive immunity, granuloma

\section{INTRODUCTION}

Tuberculosis (TB), caused by Mycobacterium tuberculosis (Mtb) infection, was among the top 10 causes of death worldwide in 2017 with about 1.5 million registered deceases (1). Mtb was responsible for approximately 10.0 million incident cases of TB disease with $10 \%$ of these occurring among children (1). One to five bacilli may suffice to transmit the infection by air (2). When inhaled, Mtb encounters a first line of defense consisting of airway epithelial cells (AECs) and "professional" phagocytes (neutrophils, monocytes and dendritic cells) $(3,4)$. If this first line succeeds in eliminating the Mtb rapidly, the infection aborts (5). Otherwise, phagocytes are infected and the Mtb reproduces inside the cells, initially causing few, if any, clinical manifestations (5). The establishment of the infection, the development of active TB (ATB) rather than latent TB infection (LTBI) and the eventual evolution of LTBI to ATB depends on the complex relation between bacterial and host factors.

The aim of this narrative minireview is to give a hint of the complexity of the abovementioned determinants and to briefly summarize the major defense mechanisms of innate and adaptive immunity against Mtb outlining the role of the different cell populations and their complex interplay. 


\section{METHODS}

In order to perform a narrative review of the available literature, we searched the PubMed database from April 2014 through April 2019, using the following key words: "immune," "immunity," "tuberculosis," "Mycobacterium tuberculosis." Subsequently, for each topic, specific key words ("susceptibility," "resistance," "virulence," "airway epithelial cell," "macrophage," "neutrophil," "dendritic cell," "natural killer," "mast cell," "complement," "CD4," “CD8," "humoral," “antibody," and "granuloma”) were associated with the word "tuberculosis" in order to access proper specific literature. The search and the selection process were not systematic. Articles were limited to English language and full text availability, and they were excluded if they were redundant or not pertinent. References of all relevant articles were also evaluated, and studies published previously than 2014 were cited if considered relevant. Results were critically summarized in the following paragraphs: (1) "host and bacterial determinants in human tuberculosis," (2) "innate immune response against Mycobacterium tuberculosis," and (3) "adaptive immune response against Mycobacterium tuberculosis.”

\section{HOST AND BACTERIAL DETERMINANTS IN HUMAN TUBERCULOSIS}

Several epidemiological models of family members who have long shared the bedroom with subjects with ATB, sailors who lived in confined spaces with subjects with open TB and extensive case studies of South African miners and Norwegian or American students, have clearly demonstrated that 5 to $20 \%$ of those who meet subjects with ATB do not become infected (resilient individuals or resisters), or become infected only transiently and then get rid of the infection (early sterilization or early clearance) (6). An individual can be defined resilient if after close and prolonged contact with the index case shows simultaneous negativity of the skin reactivity test and of the IFN $-\gamma$ release assay (IGRA) which persists for at least 1 year. Studies carried out on siblings have shown that $\mathrm{Mtb}$ resilience is more frequent between two siblings than between two unrelated subjects, suggesting the role of genetics in the development of Mtb resilience (7). Genome wide linkage analysis detected several loci like $2 q 21$ $2 q 24,5 p 13-5 q 22$, and the TST1 on $11 p 14$ associated with the resilient phenotype $(8,9)$.

On the other side, the study of TB susceptibility, has shed light onto various components of immunity to mycobacteria in humans. Different genetic polymorphisms which modulate the host immune response in favor of $\mathrm{TB}$ infection and disease progression have been identified in human leukocyte antigens (HLA), toll like receptors (TLR), vitamin D receptors (VDR), cytokines with their receptors and many other functional immune components $(10,11)$. Moreover, mendelian susceptibilities to mycobacterial disease (MSMD) have been identified as clinical conditions with selective susceptibility to poorly virulent mycobacteria in the absence of patent immunodeficiency (12). Since 1996, 11 genes which underlie
21 different genetic disorders related to interferon (IFN)- $\gamma$ immunity and responsible for MSMD have been identified (12).

Furthermore, transcriptomic studies have described a TB signature of neutrophil-driven IFN-inducible genes in ATB, including IFN- $\gamma$ but also type I IFNs, reflecting disease extension and response to treatment and highlighting the previously under-appreciated role of IFN $\alpha \beta$ signaling in TB pathogenesis $(4,13,14)$.

Beyond host factors, bacterial virulence constitutes the other major player when evaluating the risk of TB infection. Virulence is not merely limited to bacterial strain or burden in respiratory secretion but takes into account the differential Mtb gene expression in the different phases of infection. Mtb lacks classical virulence factors such as toxins and its immuneescaping ability depends on the modulation of lipid metabolism, metal-transporter proteins, protease, proteins inhibiting the antimicrobial effectors of macrophages (MФs) and many others (15).

The study of immune response in resilient and susceptible individuals, together with bacterial factors, has offered fundamental information for the understanding of TB immunology suggesting potential improvements in diagnostic and therapeutic approaches (Table 1).

\section{INNATE IMMUNE RESPONSE AGAINST Mycobacterium tuberculosis}

The significance of innate immunity in the defense against Mtb stands out clearly as we consider the MSMD where a disruption of the innate axis leads to dramatic, life-threatening clinical presentation of TB (12).

$\mathrm{M} \Phi$, neutrophils, dendritic cells (DCs), natural killer cells (NK), mast cells and complement are the major players of innate immunity. On the other hand, AECs also contribute to the defense attempt against Mtb and could be considered as innate immunity components (Table $\mathbf{1}$ ).

\section{Airway Epithelial Cells}

AECs are the first cells to come in contact with Mtb. Beyond their major role as physical barriers, they display several immunological functions albeit being traditionally considered as "non-professional" immune cells. Through pattern recognition receptors (PRRs), AECs can perceive the presence of Mtb and consequently modulate the composition of the airways surface liquid improving its antimicrobial capacity (16). Moreover, PRRs activation leads to the production of inflammatory cytokines and to the activation of mucosal-associated invariant $\mathrm{T}$ cells stimulating IFN- $\gamma$ and tumor necrosis factor (TNF)- $\alpha$ production (17).

\section{Macrophages}

$M \Phi s$ are the first line of defense, but only if the ratio of forces lies clearly to their advantage and the intervention is immediate they can cancel the infection $(5,18)$. Otherwise, they favor its development because they become first a niche for the slow replication of the Mtb and then the sanctuary for the persistence of the infection inside the phagosome during the 
TABLE 1 | Key literature of the present review.

\begin{tabular}{|c|c|c|}
\hline Paragraph & Topic & Major references \\
\hline \multirow[t]{4}{*}{$\begin{array}{l}\text { Host and bacterial } \\
\text { determinants in human } \\
\text { tuberculosis }\end{array}$} & $\begin{array}{l}\text { Host Genetic } \\
\text { polymorphism }\end{array}$ & $\begin{array}{l}\text { - Harishankar et al. (10) } \\
\text { - Casanova and Abel (7) } \\
\text { - Cobat et al. (8) } \\
\text { - Stein et al. (9) } \\
\text { - van Tong et al. (11) }\end{array}$ \\
\hline & MSMD & - Rosain et al. (12) \\
\hline & $\begin{array}{l}\text { Transcriptomic } \\
\text { studies }\end{array}$ & $\begin{array}{l}\text { - Berry et al. (13) } \\
\text { - Blankley et al. (14) }\end{array}$ \\
\hline & $\begin{array}{l}\text { Bacterial } \\
\text { Virulence factors }\end{array}$ & - Forrellad et al. (15) \\
\hline \multirow{7}{*}{$\begin{array}{l}\text { Innate immune response } \\
\text { against Mycobacterium } \\
\text { tuberculosis }\end{array}$} & AECs & $\begin{array}{l}\text { - Li et al. (16) } \\
\text { - Harriff et al. (17) }\end{array}$ \\
\hline & Macrophages & $\begin{array}{l}\text { - Queval et al. (18) } \\
\text { - Lerner et al. (19) } \\
\text { - Yuk et al. (20) } \\
\text { - Gröschel et al. (21) } \\
\text { - Bustamante et al. (22) } \\
\text { - Sia et al. (23) } \\
\text { - Sun et al. (24) } \\
\text { - Neyrolles et al. (25) } \\
\text { - Botella et al. (26) }\end{array}$ \\
\hline & Neutrophils & $\begin{array}{l}\text { - Kroon et al. (27) } \\
\text { - Lowe et al. (28) } \\
\text { - Tan et al. (29) } \\
\text { - Zhang et al. (30) }\end{array}$ \\
\hline & Dendritic cells & $\begin{array}{l}\text { - Mihret (31) } \\
\text { - Khan et al. (32) } \\
\text { - Wu et al. (33) } \\
\text { - Balboa et al. (34) } \\
\text { - Georgieva et al. (35) } \\
\text { - Velasquez et al. (36) } \\
\text { - Ehlers (37) }\end{array}$ \\
\hline & NK cells & $\begin{array}{l}\text { - Esin and Batoni (38) } \\
\text { - Arora et al. (39) } \\
\text { - Zhang et al. (40) }\end{array}$ \\
\hline & Mast Cells & $\begin{array}{l}\text { - Garcia-Rodriguez et al. (41) } \\
\text { - Carlos et al. (42) }\end{array}$ \\
\hline & Complement & $\begin{array}{l}\text { - Lubbers et al. (43) } \\
\text { - Cai et al. (44) }\end{array}$ \\
\hline \multirow[t]{4}{*}{$\begin{array}{l}\text { Adaptive immune response } \\
\text { against Mycobacterium } \\
\text { tuberculosis }\end{array}$} & $\begin{array}{l}\mathrm{CD}^{+}{ }^{+} \mathrm{T} \\
\text { lymphocytes }\end{array}$ & $\begin{array}{l}\text { - Cooper (45) } \\
\text { - Sia et al. (46) } \\
\text { - Domingo-Gonzalez et al. (47) } \\
\text { - Parkash et al. (48) } \\
\text { - Sallin et al. (49) }\end{array}$ \\
\hline & $\begin{array}{l}\text { CD8 }+\mathrm{T} \\
\text { lymphocytes }\end{array}$ & $\begin{array}{l}\text { - Lin and Flynn (50) } \\
\text { - Canaday et al. (51) } \\
\text { - Oddo et al. (52) }\end{array}$ \\
\hline & $\begin{array}{l}\text { Humoral } \\
\text { immunity }\end{array}$ & $\begin{array}{l}\text { - Kozakiewicz et al. (53) } \\
\text { - Jacobs et al. (54) } \\
\text { - Glatman-Freedman } \\
\text { and Casadevall (55) } \\
\text { - Lu et al. (56) }\end{array}$ \\
\hline & Granuloma & $\begin{array}{l}\text { - Ramakrishnan (57) } \\
\text { - Russell (58) } \\
\text { - Reece and Kaufmann (59) } \\
\text { - Refai et al. (60) } \\
\text { - Martinot (61) } \\
\text { - Russell et al. (62) }\end{array}$ \\
\hline
\end{tabular}

The major references are grouped basing on the different topics addressed in each paragraph. The most relevant and comprehensive review or study for each topic is highlighted in bold. MSMD, Mendelian Susceptibility to Mycobacterial Disease; AEC, Airway Epithelial Cell; NK, Natural Killer cells. latent infection phase. Mtb expresses an extremely wide variety of virulence factors that counteract $M \Phi$ s efforts in suppressing the pathogen. Among Mtb strategies we can include the inhibition of intracellular trafficking, the inhibition of autophagy, the acquisition of cytosol access, the induction of host cell death and the neutralization of toxic components as reactive oxygen species and toxic metals (19).

Whilst IFN- $\gamma$ is a key element in the containment of Mtb within the $M \Phi$, it is now widely recognized that performing this function requires the presence of vitamin D (63). Thanks to vitamin $\mathrm{D}$, the macrophage increases phagosome maturation and the production of antimicrobial peptides through the maximal regulation of the hCAP-18 gene encoding for cathelicidin antimicrobial peptide which activates, in turn, the transcription of autophagy-related genes (20). The $6 \mathrm{kDa}$ early secretory antigenic target (ESAT-6) protein family secretion (ESX) system is a sophisticated secretion system that Mtb uses to export proteins with immune-escaping activity. So that while the IFN- $\gamma$ axis is struggling against the ESX-system to enhance phagolysosmal activity, vitamin D deficiency abets the Mtb replication (21).

Nitric oxide (NO) within macrophages plays a less important role in humans than that one observed in animal models (19). Although, in humans too, reactive oxygen species (ROS) play a well-documented role in the immune response to $\mathrm{Mtb}$ as highlighted by the discovery of $\mathrm{TB}$ susceptibility in patients displaying mutations in a catalytic subunit of NADPH-oxidase 2 involved in ROS production on phagolysosomal membrane $(22,23)$. Moreover, it is demonstrated that Mtb affects NADPHoxidase activity through nucleoside diphosphate kinase (Npk) interaction with small GTPases involved in NADPH-oxidase assembly and functioning (24).

The fight unfolds inside the phagosome of the $М \Phi$ between the cell and the Mtb with metals as a battlefield of sorts (25). The $M \Phi$ delivers an overload of copper and zinc, which are toxic to Mtb at high concentrations. Mtb deploys a series of protection mechanisms that include controlling the capture of such metals, oxidation, and an increase in efflux (25). The up-regulation of ctpC gene encoding for the P-type ATPase which regulates the intra-bacterial levels of Zinc is a clear example of how Mtb manages to prevent heavy metal poisoning (26). As a countermove, the $M \Phi$ then attempts to block the arrival of nutrients to the Mtb such as iron and manganese (25).

\section{Neutrophils}

Neutrophil granulocytes are the most widely present cell population within BAL and sputum in patients with active TB (27). There is evidence of their role as defense mechanisms against Mtb. In particular, there is a clear inverse correlation between the number of neutrophilic granulocytes in the peripheral blood and the hazard of developing TB after contact with an infectious subject. Antimicrobial peptides and apoptotic neutrophils are phagocytized by $\mathrm{M} \Phi$ and carry out an effective activity against Mtb inside these cells (28). This is possible thanks to the fusion, within the $M \Phi$, of neutrophil granules with phagosomes containing Mtb (29). Furthermore, ETosis, extracellular traps (ET) formation, is a type of cell 
death that differently from apoptosis is characterized by DNA release, consequent $M \Phi$ activation and the formation of a DNA scaffold that incorporates pathogens and exposes them to antimicrobial molecules (64). The formation of neutrophils ETs, thus constitutes an improved killing strategy and a synergic alliance between phagocytes.

Moreover, as with many immune mechanisms, neutrophils do not only play a positive role, but can eventually constitute a negative element, causing tissue damage through production and subsequent release of their antimicrobial products (27). To this phenomenon, it must be added the potentially negative interaction with lymphocytes. Neutrophils express on their cell membrane the ligand 1 of cell death (programmed death ligand 1 or PD-L1), which interacts with the lymphocyte receptor for programmed death (programmed death receptor or PD-1), and determines, in the course of chronic infections, the loss of function and finally the death of lymphocytes (30).

Neutrophils with expressed PD-L1 are present in high proportion in patients with ATB.

\section{Dendritic Cells}

DCs are functionally located in the middle between innate and adaptive immunity. These cells play a fundamental role in the immune defense system due to antigen presentation, costimulating activity and the large cytokine production capacity with activity on the lymphocytes cluster of differentiation (CD) 4 (32). DCs role in TB immunity is controversial. Present evidence is not sufficient to establish whether these cells strengthen cellular immunity or if their manipulation by the Mtb can be used as a tool to diminish specific T-cell response (31). DCs soon become a niche for the Mtb. CD209, also called DCspecific intercellular adhesion molecule 3-grabbing non-integrin receptor (DC-SIGN), represents the gateway of $\mathrm{Mtb}$ into the DC (31). CD209 is, under normal conditions, a receptor for CD54, the intercellular adhesion molecule 1 (ICAM1) present on endothelial cells where it favors DCs migration. CD209 is coupled with the lipoarabinomannan mannose (ManLAM) of the Mtb that penetrates into the cell. This penetration leads to a disruption of DCs activity by prompting the production of interleukin (IL)-10 and reducing the production of IL-12, thus causing a suppression of $\mathrm{T}$ lymphocytes activity $(33,34)$. The manipulation of the maturation of the DCs probably represents one of the winning strategies of $\mathrm{Mbt}$ that, by restraining the activity of DCs and, consequently, of T lymphocytes, allows the Mtb, whose speed of growth is relatively slow, to efficiently establish a bridgehead in the airways (35). Based on the above mentioned mechanism DC-SIGN has recently been proposed as a potential target for a vaccine purpose eventually able to enhance immunity against Mtb (36). On the other side, DCSIGN may prevent tissue pathology by maintaining a balanced inflammatory state and thus promoting host protection (37).

\section{Natural Killer Cells}

It is certain that NK cells enter the immunological circuit of Mtb infection both in their CD56 diminished phenotype (preferential cytotoxic activity) and in CD56 bright phenotype (preferential cytokines secreting activity) (38). In several studies the percentage representation of NK cell is augmented in the peripheral blood of patients with ATB (65). There is a direct relationship between NK cell representation, clinical condition and response to therapy $(38,65)$. Nonetheless, it has not yet been ascertained exactly what the cause and the consequence is (38).

Several components of the Mbt wall are recognized and bound by the NKp44 receptor of NK cells (39). In addition, Mtbinfected NKs lyse and stimulate MФs to produce IFN- $\gamma$ and IL22, which increase phagolysosomal fusion thus inhibiting Mtb replication and stimulate the production of additional IFN- $\gamma$ by $\mathrm{CD} 8^{+}$lymphocytes. This effect is mediated by the IL-15 and IL18 production by an infected $\mathrm{M} \Phi$. As a further infection control mechanism DCs favor the development of T lymphocytes with $\gamma \delta$ receptor through TNF- $\alpha$ and IL-12 production (40).

\section{Mast Cells}

The role of mast cells in Mtb infection is not well-known in humans (41). In mice, mast cells capture Mtb via CD48 and internalize it. This process ensues the development of a cytokine cascade, some of them with protective roles, including IL-12, IL-13, IL-6, CXLL2, CCL7, CCL2, TNF- $\alpha$, and consequent neutrophils recall in the site of infection (41). Histamine's role is ambivalent in terms of Mtb clearance as on one hand it augments lung neutrophilia but on the other it seems to impair the efficient production of a $\mathrm{T}$ helper 1 (Th1) response (42). The presence of mast cell ETs containing Mtb in humans has not been proved. However, mast cells enclose a large number of mediators known to take part in the process (41).

\section{Complement Proteins}

The role of the complement cascade on the progression of the infection and Mtb disease is almost unknown (44). It is likely that the $\mathrm{C} 5$ and $\mathrm{C} 7$ components play a defensive role. However, it has been observed that a high expression of C1q correlates with a worse clinical condition, so as to be a marker between latent $\mathrm{TB}$ and active TB but still with unclear significance in terms of pathogenesis $(43,44)$.

\section{ADAPTIVE IMMUNE RESPONSE AGAINST Mycobacterium tuberculosis}

The immune response of T lymphocytes begins at the moment that Mtb spreads inside the lymph nodes but its arousal lays in the early activation of the innate immune system. Inside the lymph nodes, $\mathrm{T}$ lymphocytes undergo a process of activation and expansion of the specific populations for the Mtb antigens. However, at this point, the largest part is done and the infection is now established. Cellular immune response can be evidenced 2-6 weeks after Mtb infection by the development of a delayed hypersensitivity response to intradermal injected tuberculin (DHT) or purified protein derivative. It is important to underline that protective response to $\mathrm{TB}$ does not relate with DHT positivity and disease can occur in those who mount adequate DHT response (66) (Table 1). 


\section{Lymphocytes T CD4 ${ }^{+}$}

The in vivo human model of $\mathrm{HIV}$-infected $\mathrm{CD} 4^{+}$-depleted patients is the most striking evidence of the pivotal role of these cells in TB immunity. The process of maturation of the phagosome of $M \Phi$ is facilitated and increased by IFN- $\gamma$, the production of which is mostly dependent on the Tlymphocytes $\mathrm{CD}^{+}$with a minor support of lymphocytes $\mathrm{CD} 8^{+}$and $\mathrm{T}$ lymphocytes with $\gamma \delta$ receptor (45). Animal models of knockout mice for IFN- $\gamma$ clearly show that these animals suffer a very severe course of Mtb infection exactly as it happens in humans with MSMD. It is well known that patients with mutations in genes encoding IFN- $\gamma$ or its receptors undergo disseminated infection by BCG or other non-tuberculous components of the mycobacteria genus (12). IFN- $\gamma$ production is modest in patients with active $\mathrm{TB}$, but recovers with antitubercular treatment without reaching levels similar to those of uninfected subjects.

The optimal production of IFN- $\gamma$, as well as that of IL-17 (67), is linked to an equally optimal cooperation between DCs and T lymphocytes $\mathrm{CD} 4^{+}$. In its defensive strategy, Mtb markedly interferes in the CD40-CD40 ligand binding, that is essential for the cooperation between both cell lines (46). The importance of IFN- $\gamma$ production by $\mathrm{CD}^{+}{ }^{+}$cells is particularly relevant at the early stages of Mtb infection as it is demonstrated that adequate IFN- $\gamma$ levels can be obtained with 3 weeks of delay even in CD4disrupted mice thanks to the compensation offered by other cell types like $\mathrm{CD}^{+}(68)$.

Moreover, IFN- $\gamma$ cannot control infection alone and it requires the association of other molecules such as IL-6, IL-1 and the TNF- $\alpha$. The chemokines CCL5, CCL9, CXCL10, and CCL2 attract immunity cells at the site of infection and their production is stimulated by TNF- $\alpha$ and boosts the production of NO by MФ (47).

Several studies, both in adult and pediatric patients, have demonstrated $\mathrm{CD} 4^{+}$percentage and absolute value reduction in the peripheral blood of patients with ATB suggesting both an augmented pooling in the site of infection but also eventually a primary role of TB in immune modifications related with the severity of infection (65).

A portion of T lymphocytes are Foxp $3^{+}$and perform a control function over the activity of other $\mathrm{T}$ lymphocytes in fact, they are defined as $\mathrm{T}$ regulators (Treg). It is only on a hypothetical level that we can imagine any positive role of this cell line on the disease progression limiting tissue damage by other immune cells; however, it has been ascertained that, by restraining the response of the $\mathrm{T}$ lymphocytes, the Tregs favor the infection development and persistence (48). Similarly, T lymphocytes $\mathrm{CD}^{+}$may deal more damage, or at least become irrelevant, rather than hinder the progress of the infection (49).

\section{Lymphocytes T CD8 ${ }^{+}$}

For a long time, it was considered that, unlike $\mathrm{T}$ lymphocytes $\mathrm{CD}^{+}{ }^{+}, \mathrm{T}$ lymphocytes $\mathrm{CD}^{+}$had no role in controlling the infection and Mtb disease. This concept stemmed from the modest availability of human models with $\mathrm{T}$ lymphocyte $\mathrm{CD}^{+}$defect, unlike the large human model of HIV infection. An activity against $\mathrm{Mtb}$ is conceivable considering that $\mathrm{T}$ lymphocytes $\mathrm{CD}^{+}$recognize $\mathrm{Mtb}$ antigens through class $\mathrm{I}$ molecules of the major histocompatibility complex (MHC), and produce IL-2, IFN- $\gamma$ and TNF- $\alpha$, which have a well-known role in controlling Mtb. Furthermore, T lymphocytes $\mathrm{CD}^{+}$exert a cytolytic action against Mtb by means of perforin and granulysin, albeit not by Fas (CD95) -Fas ligand $(50,51)$ interaction. This direct cell-to-cell contact determines the apoptosis of the Mtbinfected cell (especially $M \Phi$ ) depriving Mtb from its natural growth environment and at the same time reducing its viability by unknown mechanism (52). On the other hand, lymphocytes CD ${ }^{+}$produce IL-10 and TGF- $\beta$ which instead favor the development of the Mtb infection.

\section{Humoral Adaptive Immunity}

The role of humoral adaptive immunity in TB is extremely uncertain $(53,54)$. Complement-mediated opsonization does not alter Mtb survival. High levels of antibody titers correlate with more serious conditions of infection and disease, and passive immunization with antibodies does not confer protection (55). Patients with a defective antibody-production mechanism and/or B lymphocyte defect are not particularly at risk of TB infection.

The role of the crystallizable fragment or Fc in the constant portion of the immunoglobulin, which binds and activates various cell lines present in the granuloma (NK cells, monocytes, neutrophils) the low-affinity Fc $\gamma$ RIIIb receptor and the high affinity Fc $\gamma$ RIIa receptor have shown different functional profiles and glycosylation patterns in subjects with ATB rather than LTBI $(54,56)$.

The loss of Fc $\gamma$ RIIIb activity and the increase of Fc $\gamma$ RIIamediated inhibitory function (which correlates with a high IL10 production) are associated with a worse clinical profile and can distinguish ATB from LTBI and suggests a role of antibodies in the augmented phagolysosomal maturation and Mtb killing observed in LTBI patients (56).

\section{The Ancestral Defense: Granuloma}

Following the development of adaptive immunity, a complex and well-coordinated mechanism is established between both immunity mechanisms, i.e., innate and adaptive, which seal the Mtb inside granulomas $(5,58,59)$. This mechanism develops in at least $90 \%$ of the infected subjects and leads to LTBI. During latent TB, which would be better described as nonreplicating-persistence phase (in fact, Mtb works perfectly albeit in a different way than during active TB), the subject is generally positive for the tuberculin skin test and for the IGRA (69). Latent TB becomes active when, for the most various reasons, a condition of immunodepression develops. At this stage, the subject may become capable of transmitting the infection because the granuloma opens in the bronchial lumen and Mtb are expelled when coughing. At the beginning of the infection, Mtb demands an environment with inflammatory traits to develop the granuloma; subsequently, however, its survival is linked to an environment lacking or with low inflammation. This switch is caused by ESAT-6 (60), a well-known Mtb virulence factor involved in the ESX secretion system, to which it gives its name. ESAT- 6 causes the transformation of $M \Phi$ from phenotype M1, which produces IL-6, IL-12 and TNF- $\alpha$, into M $\Phi$ with phenotype M2, which is capable of stimulating production of 
IL-10 (60, 61, 70). As currently known, IL-6 and TNF- $\alpha$ favor inflammation, whilst IL-10 curbs it. Accordingly, the formation of the granuloma is triggered by the $M \Phi$ and then develops with multi-nucleated giant cells and $M \Phi$ with abundant presence of intracytoplasmic lipids, which lend these cells their frothy appearance. Around these cells, there is a ring of T lymphocytes although B lymphocytes, neutrophils and dendritic cells (CD) also participate in the formation of granuloma (4).

Inside the granuloma, cholesterol-and not glucose or glycerol-is the only carbon source. This leads to a lack of carbon and nutrients, hypoxia and a high concentration of nitric oxide (NO). The significance of cholesterol in the survival of Mtb inside the granuloma is evidenced by the negative role that statins play against Mtb $(61,70)$.

The debate remains open on whether the granuloma is purely protective for the host or if it promotes disease progression and tissue damage (4). This uncertainty depends on the extreme heterogeneity detected in granuloma morphology at the different stages of disease, on the role of inflammation, hypoxia and differential Mtb gene expression and lipid metabolism manipulation inside the granulomas of ATB and LTBI patients (62). The most likely answer is that an homeostatic interaction establishes and the granuloma becomes a well-suited shelter for both Mtb long-term survival and host protection (57).

\section{DISCUSSION}

\section{Mycobacterium tuberculosis: The Great Manipulator}

The different cell lines of innate and adaptive immunity come into play at different times in the battle against Mtb in a clash in which the genetic susceptibility of the host and the virulence of the pathogen play decisive roles for the final outcome. The success of $\mathrm{Mtb}$ over thousands of years against man arises from its extraordinary ability to subvert the mechanisms that should eliminate it in the $M \Phi$ from the infection onset. At the onset of the infection, Mtb manages to perforate the phagosome in the M $\Phi$ through the ESX system and, therefore, to block its maturation via $\mathrm{Npk}$, which inhibits lysosomal traffic and

\section{REFERENCES}

1. WHO. Global Tuberculosis Report. (2018). Available online at: http://www. who.int/tb/publications/global_report/en/ (accessed June 11, 2019).

2. Tellier R, Li Y, Cowling BJ, Tang JW. Recognition of aerosol transmission of infectious agents: a commentary. BMC Infect Dis. (2019) 19:101. doi: 10.1186/s12879-019-3707-y

3. Middleton AM, Chadwick MV, Nicholson AG, Dewar A, Groger RK, Brown EJ, et al. Interaction of Mycobacterium tuberculosis with human respiratory mucosa. Tuberculosis. (2002) 82:69-78. doi: 10.1054/tube.2002.0324

4. O'Garra A, Redford PS, McNab FW, Bloom CI, Wilkinson RJ, Berry MPR. The immune response in tuberculosis. Annu Rev Immunol. (2013) 31:475-527. doi: 10.1146/annurev-immunol-032712-095939

5. de Martino M, Galli L, Chiappini E. Reflections on the immunology of tuberculosis: will we ever unravel the skein? BMC Infect Dis. (2014) 14 (Suppl. 1):S1. doi: 10.1186/1471-2334-14-S1-S1
NADPH-oxidase activity (31). With various mechanisms, some of which also operate at the level of macrophage DNA, the Mbt prevents the activation of pathogen destruction systems, which are implemented through autophagy. The Mtb DNA manages to prevent the activation of the AIM2 inflammasome thus hindering the synthesis of IL-1 $\beta$ and IL-18 (31). Under normal conditions, IFN- $\gamma$ stimulates the expression of MHC class II molecules on the MФ. But Mtb, thanks to the prolonged activation of TLR2, succeeds in suppressing this mechanism. Even in cells that already express class II MHC molecules, Mtb manages to block the presentation of antigens by the action on ESCRT (endosomal sorting complexes required for transport) of its EsxG.EsxH protein (31). The "great manipulator" also interferes with the functions of DCs, neutrophils and all other components of the immune system.

In conclusion, having to deal with a micro-organism of great evasive abilities, immune mechanisms have only one way to go: to focus on a very rapid response at the onset of the infection.

Paraphrasing a famous aphorism by General Erwin Rommel about amphibious battles that is well-suited to TB, victory or defeat against $\mathrm{Mtb}$ is decided in the first moments of the infection (5). Exactly as it happened on the first day of the amphibious assault, the day that General Erwin Rommel defined as "the longest day." When it comes to implementing new prevention and therapeutic approaches, a clear understanding of the interplay between the immune system and $\mathrm{Mtb}$ at a molecular level is the only way to unravel this millenary skein.

\section{AUTHOR CONTRIBUTIONS}

MdM wrote the main body of this minireview. LL, LG, and EC contributed with literature search and revisions.

\section{FUNDING}

This work received no fundings. The payment of fees for open access publication will be supported by the Dipartimento di Medicina Sperimentale e Clinica of the University of Florence.

6. Ringshausen FC, Nienhaus A, Schablon A, Schlösser S, Schultze-Werninghaus G, Rohde G. Predictors of persistently positive Mycobacterium-tuberculosisspecific interferon-gamma responses in the serial testing of health care workers. BMC Infect Dis. (2010) 10:220. doi: 10.1186/1471-2334-10-220

7. Casanova J-L, Abel L. Genetic dissection of immunity to mycobacteria: the human model. Annu Rev Immunol. (2002) 20:581-620. doi: 10.1146/annurev.immunol.20.081501.125851

8. Cobat A, Gallant CJ, Simkin L, Black GF, Stanley K, Hughes J, et al. Two loci control tuberculin skin test reactivity in an area hyperendemic for tuberculosis. J Exp Med. (2009) 206:2583-91. doi: 10.1084/jem.20090892

9. Stein CM, Zalwango S, Malone LL, Won S, Mayanja-Kizza H, Mugerwa RD, et al. Genome scan of M. tuberculosis infection and disease in Ugandans. PLoS ONE. (2008) 3:e4094. doi: 10.1371/journal.pone.0004094

10. Harishankar M, Selvaraj P, Bethunaickan R. Influence of genetic polymorphism towards pulmonary tuberculosis susceptibility. Front Med. (2018) 5:213. doi: 10.3389/fmed.2018.00213 
11. van Tong $\mathrm{H}$, Velavan TP, Thye T, Meyer CG. Human genetic factors in tuberculosis: an update. Trop Med Int Health. (2017) 22:1063-71. doi: 10.1111/tmi.12923

12. Rosain J, Kong X-F, Martinez-Barricarte R, Oleaga-Quintas C, Ramirez-Alejo N, Markle J, et al. Mendelian susceptibility to mycobacterial disease: 20142018 update. Immunol Cell Biol. (2019) 97:360-7. doi: 10.1111/imcb.12210

13. Berry MPR, Graham CM, McNab FW, Xu Z, Bloch SAA, Oni T, et al. An interferon-inducible neutrophil-driven blood transcriptional signature in human tuberculosis. Nature. (2010) 466:973-7. doi: 10.1038/nature09247

14. Blankley S, Graham CM, Turner J, Berry MPR, Bloom CI, Xu Z, et al. The transcriptional signature of active tuberculosis reflects symptom status in extra-pulmonary and pulmonary tuberculosis. PLOS ONE. (2016) 11:e0162220. doi: 10.1371/journal.pone.0162220

15. Forrellad MA, Klepp LI, Gioffré A, Sabio y García J, Morbidoni HR, Santangelo M de la P, et al. Virulence factors of the Mycobacterium tuberculosis complex. Virulence. (2013) 4:3-66. doi: 10.4161/viru.22329

16. Li Y, Wang Y, Liu X. The role of airway epithelial cells in response to mycobacteria infection. Clin Dev Immunol. (2012) 2012:791392. doi: $10.1155 / 2012 / 791392$

17. Harriff MJ, Cansler ME, Toren KG, Canfield ET, Kwak S, Gold MC, et al. Human lung epithelial cells contain Mycobacterium tuberculosis in a late endosomal vacuole and are efficiently recognized by CD8 ${ }^{+}$T cells. PLoS ONE. (2014) 9:e97515. doi: 10.1371/journal.pone.0097515

18. Queval CJ, Brosch R, Simeone R. The macrophage: a disputed fortress in the battle against Mycobacterium tuberculosis. Front Microbiol. (2017) 8:2284. doi: 10.3389/fmicb.2017.02284

19. Lerner TR, Borel S, Gutierrez MG. The innate immune response in human tuberculosis. Cell Microbiol. (2015) 17:1277-85. doi: 10.1111/cmi.12480

20. Yuk J-M, Shin D-M, Lee H-M, Yang C-S, Jin HS, Kim K-K, et al. Vitamin D3 induces autophagy in human monocytes/macrophages via cathelicidin. Cell Host Microbe. (2009) 6:231-43. doi: 10.1016/j.chom.2009.08.004

21. Gröschel MI, Sayes F, Simeone R, Majlessi L, Brosch R. ESX secretion systems: mycobacterial evolution to counter host immunity. Nat Rev Microbiol. (2016) 14:677-91. doi: 10.1038/nrmicro.2016.131

22. Bustamante J, Arias AA, Vogt G, Picard C, Galicia LB, Prando C, et al. Germline CYBB mutations that selectively affect macrophages in kindreds with X-linked predisposition to tuberculous mycobacterial disease. Nat Immunol. (2011) 12:213-21. doi: 10.1038/ni.1992

23. Sia JK, Georgieva M, Rengarajan J. Innate immune defenses in human tuberculosis: an overview of the interactions between Mycobacterium tuberculosis and innate immune cells. J Immunol Res. (2015) 2015:747543. doi: 10.1155/2015/747543

24. Sun J, Singh V, Lau A, Stokes RW, Obregón-Henao A, Orme IM, et al. Mycobacterium tuberculosis nucleoside diphosphate kinase inactivates small GTPases leading to evasion of innate immunity. PLoS Pathog. (2013) 9:e1003499. doi: 10.1371/journal.ppat.1003499

25. Neyrolles O, Wolschendorf F, Mitra A, Niederweis M. Mycobacteria, metals, and the macrophage. Immunol Rev. (2015) 264:249-63. doi: 10.1111/imr.12265

26. Botella H, Peyron P, Levillain F, Poincloux R, Poquet Y, Brandli $\mathrm{I}$, et al. Mycobacterial p(1)-type ATPases mediate resistance to zinc poisoning in human macrophages. Cell Host Microbe. (2011) 10:248-59. doi: 10.1016/j.chom.2011.08.006

27. Kroon EE, Coussens AK, Kinnear C, Orlova M, Möller M, Seeger A, et al. Neutrophils: innate effectors of TB resistance? Front Immunol. (2018) 9:2637.doi: 10.3389/fimmu.2018.02637

28. Lowe DM, Demaret J, Bangani N, Nakiwala JK, Goliath R, Wilkinson KA, et al. Differential effect of viable versus necrotic neutrophils on Mycobacterium tuberculosis growth and cytokine induction in whole blood. Front Immunol. (2018) 9:903. doi: 10.3389/fimmu.2018.00903

29. Tan BH, Meinken C, Bastian M, Bruns H, Legaspi A, Ochoa MT, et al. Macrophages acquire neutrophil granules for antimicrobial activity against intracellular pathogens. J Immunol. (2006) 177:1864-71. doi: 10.4049/jimmunol.177.3.1864

30. Zhang Y, Zhou Y, Lou J, Li J, Bo L, Zhu K, et al. PD-L1 blockade improves survival in experimental sepsis by inhibiting lymphocyte apoptosis and reversing monocyte dysfunction. Crit Care. (2010) 14:R220.doi: 10.1186/cc9354
31. Mihret A. The role of dendritic cells in Mycobacterium tuberculosis infection Virulence. (2012) 3:654-9. doi: 10.4161/viru.22586

32. Khan N, Vidyarthi A, Pahari S, Agrewala JN. Distinct strategies employed by dendritic cells and macrophages in restricting Mycobacterium tuberculosis infection: different philosophies but same desire. Int Rev Immunol. (2016) 35:386-98. doi: 10.3109/08830185.2015.1015718

33. Wu T, Guo S, Wang J, Li L, Xu L, Liu P, et al. Interaction between mannosylated lipoarabinomannan and dendritic cell-specific intercellular adhesion molecule-3 grabbing nonintegrin influences dendritic cells maturation and $\mathrm{T}$ cell immunity. Cell Immunol. (2011) 272:94-101. doi: 10.1016/j.cellimm.2011.09.001

34. Balboa L, Romero MM, Yokobori N, Schierloh P, Geffner L, Basile JI, et al. Mycobacterium tuberculosis impairs dendritic cell response by altering CD1b, DC-SIGN and MR profile. Immunol Cell Biol. (2010) 88:716-26. doi: 10.1038/icb.2010.22

35. Georgieva M, Sia JK, Bizzell E, Madan-Lala R, Rengarajan J. Mycobacterium tuberculosis GroEL2 modulates dendritic cell responses. Infect Immun. (2018) 86:e00387-17. doi: 10.1128/IAI.00387-17

36. Velasquez LN, Stüve P, Gentilini MV, Swallow M, Bartel J, Lycke NY, et al. Targeting Mycobacterium tuberculosis antigens to dendritic cells via the DC-specific-ICAM3-grabbing-nonintegrin receptor induces strong T-helper 1 immune responses. Front Immunol. (2018) 9:471. doi: 10.3389/fimmu.2018.00471

37. Ehlers S. DC-SIGN and mannosylated surface structures of Mycobacterium tuberculosis: a deceptive liaison. Eur J Cell Biol. (2010) 89:95-101. doi: 10.1016/j.ejcb.2009.10.004

38. Esin S, Batoni G. Natural killer cells: a coherent model for their functional role in Mycobacterium tuberculosis infection. J Innate Immun. (2015) 7:11-24. doi: $10.1159 / 000363321$

39. Arora P, Foster EL, Porcelli SA. CD1d and natural killer T cells in immunity to Mycobacterium tuberculosis. Adv Exp Med Biol. (2013) 783:199-223. doi: 10.1007/978-1-4614-6111-1_11

40. Zhang R, Zheng X, Li B, Wei H, Tian Z. Human NK cells positively regulate gammadelta $\mathrm{T}$ cells in response to Mycobacterium tuberculosis. J Immunol. (2006) 176:2610-6. doi: 10.4049/jimmunol.176.4.2610

41. Garcia-Rodriguez KM, Goenka A, Alonso-Rasgado MT, HernándezPando R, Bulfone-Paus S. The role of mast cells in tuberculosis: orchestrating innate immune crosstalk? Front Immunol. (2017) 8:1290. doi: 10.3389/fimmu.2017.01290

42. Carlos D, Fremond C, Samarina A, Vasseur V, Maillet I, Ramos SG, et al. Histamine plays an essential regulatory role in lung inflammation and protective immunity in the acute phase of Mycobacterium tuberculosis infection. Infect Immun. (2009) 77:5359-68. doi: 10.1128/IAI. 01497-08

43. Lubbers R, Sutherland JS, Goletti D, de Paus RA, van Moorsel CHM, Veltkamp $\mathrm{M}$, et al. Complement component $\mathrm{Clq}$ as serum biomarker to detect active tuberculosis. Front Immunol. (2018) 9:2427. doi: 10.3389/fimmu.2018.02427

44. Cai Y, Yang Q, Tang Y, Zhang M, Liu H, Zhang G, et al. Increased complement $\mathrm{C} 1 \mathrm{q}$ level marks active disease in human tuberculosis. PLoS ONE. (2014) 9:e92340. doi: 10.1371/journal.pone.0092340

45. Cooper AM. Cell-mediated immune responses in tuberculosis. Annu Rev Immunol. (2009) 27:393-422. doi: 10.1146/annurev.immunol.021908.132703

46. Sia JK, Bizzell E, Madan-Lala R, Rengarajan J. Engaging the CD40CD40L pathway augments T-helper cell responses and improves control of Mycobacterium tuberculosis infection. PLoS Pathog. (2017) 13:e1006530. doi: 10.1371/journal.ppat.1006530

47. Domingo-Gonzalez R, Prince O, Cooper A, Khader SA. Cytokines and chemokines in Mycobacterium tuberculosis infection. Microbiol Spectr. (2016) 4:TBTB2-0018-2016. doi: 10.1128/microbiolspec.TBTB2-0018-2016

48. Parkash O, Agrawal S, Madhan Kumar M. T regulatory cells: Achilles' heel of Mycobacterium tuberculosis infection? Immunol Res. (2015) 62:386-98. doi: 10.1007/s12026-015-8654-0

49. Sallin MA, Sakai S, Kauffman KD, Young HA, Zhu J, Barber DL. Th1 differentiation drives the accumulation of intravascular, nonprotective CD4 T cells during tuberculosis. Cell Rep. (2017) 18:3091-104. doi: 10.1016/j.celrep.2017.03.007

50. Lin PL, Flynn JL. CD8 T cells and Mycobacterium tuberculosis infection. Semin Immunopathol. (2015) 37:239-49. doi: 10.1007/s00281-015-0490-8 
51. Canaday DH, Wilkinson RJ, Li Q, Harding CV, Silver RF, Boom WH. CD4 ${ }^{+}$ and $\mathrm{CD}^{+} \mathrm{T}$ cells kill intracellular Mycobacterium tuberculosis by a perforin and Fas/Fas ligand-independent mechanism. J Immunol. (2001) 167:2734-42. doi: 10.4049/jimmunol.167.5.2734

52. Oddo M, Renno T, Attinger A, Bakker T, MacDonald HR, Meylan PR. Fas ligand-induced apoptosis of infected human macrophages reduces the viability of intracellular Mycobacterium tuberculosis. J Immunol. (1998) 160:5448-54.

53. Kozakiewicz L, Phuah J, Flynn J, Chan J. The role of B cells and humoral immunity in Mycobacterium tuberculosis infection. Adv Exp Med Biol. (2013) 783:225-50. doi: 10.1007/978-1-4614-6111-1_12

54. Jacobs AJ, Mongkolsapaya J, Screaton GR, McShane H, Wilkinson RJ. Antibodies and tuberculosis. Tuberculosis. (2016) 101:102-3. doi: $10.1016 /$ j.tube.2016.08.001

55. Glatman-Freedman A, Casadevall A. Serum therapy for tuberculosis revisited: reappraisal of the role of antibody-mediated immunity against Mycobacterium tuberculosis. Clin Microbiol Rev. (1998) 11:514-32.

56. Lu LL, Chung AW, Rosebrock TR, Ghebremichael M, Yu WH, Grace PS, et al. A functional role for antibodies in tuberculosis. Cell. (2016) 167:433-443.e14. doi: 10.1016/j.cell.2016.08.072

57. Ramakrishnan L. Revisiting the role of the granuloma in tuberculosis. Nat Rev Immunol. (2012) 12:352-66. doi: 10.1038/nri3211

58. Russell DG. Who puts the tubercle in tuberculosis? Nat Rev Microbiol. (2007) 5:39-47. doi: 10.1038/nrmicro1538

59. Reece ST, Kaufmann SHE. Floating between the poles of pathology and protection: can we pin down the granuloma in tuberculosis? Curr Opin Microbiol. (2012) 15:63-70. doi: 10.1016/j.mib.2011.10.006

60. Refai A, Gritli S, Barbouche M-R, Essafi M. Mycobacterium tuberculosis virulent factor ESAT-6 drives macrophage differentiation toward the proinflammatory M1 phenotype and subsequently switches it to the antiinflammatory M2 phenotype. Front Cell Infect Microbiol. (2018) 8:327. doi: $10.3389 /$ fcimb.2018.00327

61. Martinot AJ. Microbial offense vs host defense: who controls the TB granuloma? Vet Pathol. (2018) 55:14-26. doi: 10.1177/0300985817705177

62. Russell DG, Cardona P-J, Kim M-J, Allain S, Altare F. Foamy macrophages and the progression of the human tuberculosis granuloma. Nat Immunol. (2009) 10:943-8.

63. Gou X, Pan L, Tang F, Gao H, Xiao D. The association between vitamin D status and tuberculosis in children: a meta-analysis. Medicine. (2018) 97:e12179. doi: 10.1097/MD.0000000000012179
64. Guimarães-Costa AB, Nascimento MTC, Wardini AB, Pinto-daSilva LH, Saraiva EM. ETosis: a microbicidal mechanism beyond cell death. J Parasitol Res. (2012) 2012:929743. doi: 10.1155/2012/9 29743

65. Venturini E, Lodi L, Francolino I, Ricci S, Chiappini E, de Martino M, et al. CD3, CD4, CD8, CD19 and CD16/CD56 positive cells in tuberculosis infection and disease: peculiar features in children. Int J Immunopathol Pharmacol. (2019) 33:2058738419840241. doi: 10.1177/2058738419 840241

66. McKinney J, Jacobs W, Bloom B. Persisting problems in tuberculosis. In: Krause RM, editor. Emerging Infections. San Diego, CA: Academic Press (1998). p. 51-146.

67. Monin L, Griffiths KL, Slight S, Lin Y, Rangel-Moreno J, Khader SA. Immune requirements for protective Th17 recall responses to Mycobacterium tuberculosis challenge. Mucosal Immunol. (2015) 8:1099-109. doi: $10.1038 / \mathrm{mi} .2014 .136$

68. Caruso AM, Serbina N, Klein E, Triebold K, Bloom BR, Flynn JL. Mice deficient in CD4 T cells have only transiently diminished levels of IFNgamma, yet succumb to tuberculosis. J Immunol. (1999) 162:5407-16.

69. Wayne LG. Dynamics of submerged growth of Mycobacterium tuberculosis under aerobic and microaerophilic conditions. Am Rev Respir Dis. (1976) 114:807-11.

70. Gibson SER, Harrison J, Cox JAG. Modelling a silent epidemic: a review of the in vitro models of latent tuberculosis. Pathogens. (2018) 7:E88. doi: $10.3390 /$ pathogens7040088

Conflict of Interest Statement: The authors declare that the research was conducted in the absence of any commercial or financial relationships that could be construed as a potential conflict of interest.

The handling editor declared a past co-authorship with several of the authors MdM, LG, and EC.

Copyright (c) 2019 de Martino, Lodi, Galli and Chiappini. This is an open-access article distributed under the terms of the Creative Commons Attribution License (CC $B Y)$. The use, distribution or reproduction in other forums is permitted, provided the original author(s) and the copyright owner(s) are credited and that the original publication in this journal is cited, in accordance with accepted academic practice. No use, distribution or reproduction is permitted which does not comply with these terms. 